\title{
Minimally invasive, no hardware subtalar arthrodesis with autogenous posterior iliac bone graft
}

\author{
Mohamed A. M. Eid • Maged Abou El-Soud • \\ Mahmoud A. Mahran · Timour F. El-Hussieni
}

Received: 20 April 2009/Accepted: 7 February 2010/Published online: 4 March 2010

(C) Springer-Verlag 2010

\begin{abstract}
Sixteen patients underwent minimally invasive subtalar arthrodesis through a mini-invasive approach with posterior iliac graft between 2004 and 2006. No hardware was used to transfix the arthrodesis and partial weight bearing was allowed immediately. The primary indication for surgery was the squeal of fracture os calcis in terms of subtalar joint arthritis, loss of heel height, malalignment of the hindfoot, and pain with weight bearing. There were 12 male and 4 female patients with a mean age of 30 (range 17-52). Patients were followed up for a period of 40.8 months (range 36-48 months). The mean interval from injury to fusion was $2(+0.6)$ years ranging from 6 months to 6 years post fracture. The average clinical rating scale based on the American Orthopaedic Foot and Ankle Society (AOFAS) improved from 36 preoperatively to 78 at the latest follow-up $(P<0.05)$. Union rate was 94\%. Radiographic evaluation revealed a mean increase in calcaneal inclination of $6.25+8.3^{\circ}(P<0.07)$ and a mean increase in the lateral talocalcaneal angle of $7.42+10.2^{\circ}$ $(P<0.08)$. Complications were graft nonunion in 1 patient and transient tendoachilles tendinitis in another. This technique can be used to decrease the morbidity associated with the late complications of os calcis fractures by aligning the hindfoot, restoring the heel height and correcting calcaneal and talar inclination. It offers the advantage of early weight bearing while avoiding hardware complications.
\end{abstract}

Study conducted in Ain-Shams University Hospitals, Department of Orthopaedic Surgery, Ain Shams University, Cairo, Egypt.

M. A. M. Eid · M. A. El-Soud · M. A. Mahran (凹) ·

T. F. El-Hussieni

Department of Orthopaedic Surgery,

Ain Shams University, Cairo, Egypt

e-mail: mahmoudmahran@gmail.com
Keywords Subtalar arthrodesis - Os calcis fracture · Minimally invasive Posterior iliac crest bone . Grafting

\section{Introduction}

The anatomy of the calcaneum and its relation to the wedge-shaped inferior surface of the talus is such that, when it is fractured, most impact usually falls on the posterior facet of the subtalar joint. The functional outcome after such fractures depends on the residual subtalar joint distortion and secondary stiffness that develops in the foot. Osteoarthritis can develop in a damaged and distorted subtalar joint, and this complication is the main cause of late and prolonged disability [8].

When conservative management fails for symptomatic post-traumatic subtalar arthritis, arthrodesis of the hindfoot is the operative treatment of choice. Triple arthrodesis was judged to be the standard treatment by some surgeons previously but, due to possible complications, subtalar arthrodesis is now suggested as a less radical option [7, 21, 23].

Posterior subtalar joint arthrodesis using bone-block distraction has been described to address late complications of neglected, displaced intra-articular fractures of the calcaneum $[1,26]$. The pathoanatomic features of these cases include subtalar incongruity and arthritis, loss of calcaneal height, lateral wall expansion, anterior tibiotalar impingement, varus or valgus malalignment of the hindfoot, and a weakened lever arm for the Achilles tendon. Symptoms include fibular abutment with peroneal tendon and sural nerve irritation, poor propulsion, anterior ankle pain, and difficulty in fitting foot wear. The goals of the reconstructive arthrodesis procedure are restoration of heel 
height, decompression of the anterior ankle joint, elimination of subtalar arthritis, and correction of hindfoot malalignment [1, 22, 26].

Different surgical techniques have been proposed to achieve these surgical aims. The surgical approach, source of bone graft, type of internal fixation, and postoperative regimen have differed among several authors. A surgical technique that is less traumatic, minimizes the soft-tissue complications of hindfoot surgery, has few or no hardware complications, and that uses an optimal bone graft source is still awaited.

The aim of this current study is to investigate the efficacy of a minimally invasive posterolateral percutaneous approach for subtalar arthrodesis using posterior iliac bone graft, with immediate weight bearing in a cast boot, for such cases of neglected or malreduced calcaneal fractures.

\section{Patients and methods}

Sixteen patients with sequelae from os calcis fractures were treated by a minimally invasive subtalar fusion technique through a percutaneous posterolateral approach using posterior iliac bone graft. All patients presented with complaints of pain that limited their activities of daily living. There were 12 male and 4 female patients with a mean age of 30 (range 17-52). All operations were performed by the authors between 2004 and 2006. The inclusion criteria for the sample in this study were that patients had:

1. Calcaneal fractures that had been managed conservatively in the first instance.

2. Pain that limited activities of daily living and which had persisted for a minimum of 6 months post injury.

3. The subtalar joint identified as the source of the pain.

The following patients were excluded:

1. Patients with symptomatic calcaneocuboid subluxation or arthritis.

2. Pain from lateral calcaneal wall expansion and peroneal tendon irritation as confirmed through a diagnostic local anaesthetic test.

3. Those with severe varus malalignment of the heel.

The study was approved by the local ethics committee and all patients gave their informed consent prior to their inclusion in the study.

Preoperative evaluation

A careful history and physical examination was performed to assess for problems after the calcaneal fracture and identify the cause of pain. Radiographs were obtained and preoperative scores using the AOFAS (American
Orthopaedic Foot and Ankle Surgery) scale was documented for every case.

Operative technique

With the patient prone, the posterior subtalar joint is exposed through a $3-\mathrm{cm}$ vertical incision placed between the lateral malleolus and the Achilles tendon (Fig. 1a). With blunt dissection, the sural nerve is identified, retracted laterally, and protected with a blunt retractor during the procedure. Conventional dynamic hip screw (DHS) insertion instruments are used to create a fusion tunnel traversing the joint from posterior to anterior. Under fluoroscopic visualization, a guide wire is advanced through the posterior subtalar joint, traversing the sinus tarsi, and up to the level of the midtarsal joint (Fig. 1b). The 8-mm DHS core reamer is then used to ream the whole tract along the guide wire (Fig. 1c). Correction of hindfoot varus or valgus is possible by altering the tunnel placement medially or laterally, respectively. The assistant harvests a cortico-cancellous wedge from the posterior iliac crest measuring $2.5 \mathrm{~cm}$ by $1 \mathrm{~cm}$ by $1 \mathrm{~cm}$, while residual cartilage is denuded from the tunnel using a curette, and the subchondral surfaces are fish-scaled with a small osteotome to increase the fusion surface area. The graft is then impacted in the tunnel until the posterior edge of the graft is flush with the posterior limit of the joint (Fig. 1d-f). In patients with increased plantar-flexion of the os calcis (decreased calcaneal pitch angle preoperatively), the graft would be fashioned into a more conical shape, with a broader posterior base and a narrower anterior apex so as to create greater distraction posteriorly and improve the calcaneal pitch. The wound is closed and a cast boot is applied allowing immediate weight bearing on the second postoperative day. The idea of using the DHS core reamer in creating the fusion tunnel was based on studying sagittal sections of the foot from the Visible Human Project; we estimated an 8-mm reamer would be able to cut a safe and useful fusion tunnel (Fig. 1g, h).

\section{Postoperative management}

All patients were allowed up on crutches bearing partial weight on the operated limb in a cast boot for 2 weeks, and then full weight bearing till the cast boot was removed at 6 weeks postoperatively. The patients were usually discharged after $1-2$ days (mean $1.3 \pm 0.4$ ). Postsurgery evaluation was carried out at regular intervals of 2,6 , and 10 weeks and then every 3 months as necessary.

Clinical evaluation

A careful history and physical examination was performed with particular attention to wound-healing, infection, 

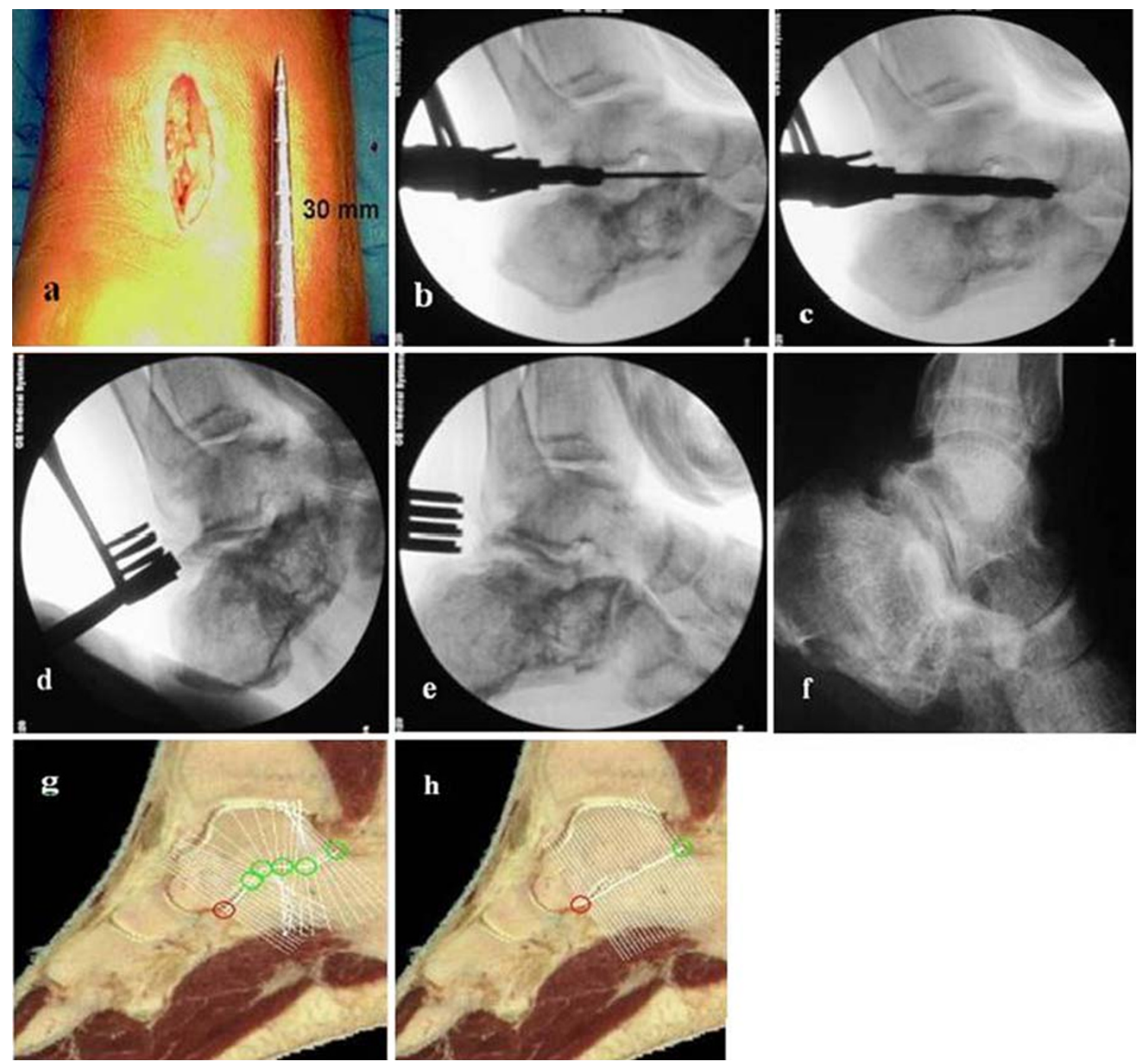

Fig. 1 Operative technique: a Surgical approach, b guide wire placement, $\mathbf{c}$ reaming the fusion tunnel using a conventional DHS core reamer, $\mathbf{d}$ graft impaction, $\mathbf{e}$ final graft position, $\mathbf{f}$ postoperative

radiograph, $\mathbf{g}$ fusion pathway delineated by $6-\mathrm{mm}$ circles, and $\mathbf{h}$ straight path of the 8-mm DHS core reamer

2. Can negotiate with discomfort or instability.

3. Can negotiate with pain.

4. Cannot negotiate or avoid uneven ground. resolved), hindfoot alignment, subfibular impingement, range of motion, pain at the ankle or the transverse tarsal joint, and sural nerve symptoms. The ankle-hindfoot scale of the American Orthopaedic Foot and Ankle Society (AOFAS scale) was used to assess functional outcome and compared to the preoperative score. Special emphasis was made on the ability to negotiate uneven ground during walking as this aspect is most relevant in third world countries where the different nature of streets required this ability to be preserved.

A modified scale (1-4) was created for the ability to negotiate uneven ground:

1. Can negotiate uneven ground.

\section{Radiographic evaluation}

Preoperative and postoperative radiographic evaluation consisted of lateral and anteroposterior radiographs of the foot, two Broden radiographs of the hindfoot, and an anteroposterior radiograph of the ankle. Union was determined on the basis of the lateral radiograph and the two Broden radiographs. The presence of arthritic joints adjacent was from all five views.

The calcaneal pitch determines the calcaneal inclination; this angle is formed by plantar border of the calcaneum to 
the inferior border of the sole or film in a weight bearing view. The lateral talocalcaneal angle is formed by the bisection of the talar axis and plantar border of the calcaneum (normal, $25-45^{\circ}$ ). Significant differences were determined using a paired sample $t$ test with $P<0.05$ taken as the level of significance. Complete union was determined through radiographs showing complete bridging with the absence of radiolucencies at both the grafttalus and graft-calcaneum interfaces and absence of pain on clinical stress-testing.

Patients without adequate confirmation of clinical and radiographic healing were followed at monthly intervals until either healing occurred or delayed union or nonunion problems were declared.

\section{Results}

The current study included 12 males and 4 females with a mean age of $30( \pm 9.3)$. All had previous os calcis fractures that was deemed nonreconstructable and managed conservatively. There were 11 right feet and 5 left feet in the sample. The mean interval from injury to fusion was 2 years $( \pm 0.6)$ ranging from 6 months to 6 years. The mean follow-up period was $40.8( \pm 5.4)$ months.

The American Orthopaedic Foot and Ankle Society scaling system [17] was used to evaluate the clinical results (Table 1). Of the 16 patients included in this study, 10 patients had occasional pain on high activity, 5 patients had mild pain on moderate activity, and only 1 patient had daily moderate daily pain on low activity. That patient was diagnosed with transient Achilles tendonitis in the early postoperative course. None of the patients had severe or constant pain. Twelve patients had no significant limitation of daily or recreational activities, 3 patients had significant limitation of recreational but not daily activities, and 1 patient had limitation of both. All our patients walked unaided except one who used a walking stick occasionally. None of our patients had restrictions to footwear. Fourteen patients were able to walk further than 6 blocks, and two patients were able to walk between 4 and 6 blocks.

Eight patients had no difficulty on any walking surface and were able to negotiate uneven ground. Seven patients had mild discomfort and/or instability in negotiating uneven ground and one patient who had transient Achilles tendonitis could only negotiate uneven ground with pain. The ankle to hindfoot alignment was good in 14 patients and fair in 2 patients who walked with a slight limp. However, no other gait abnormality was noted in all the other patients at their latest follow-up. The mean tibiocalcaneal angle was $9 \pm 5^{\circ}$ of valgus on the involved side and $8 \pm 2^{\circ}$ of valgus on the uninvolved side. The mean dorsiflexion range was $7 \pm 4^{\circ}$ before and $9 \pm 5^{\circ}$ after
Table 1 The American Orthopaedic Foot and Ankle Society scoring system

\begin{tabular}{lr}
\hline Pain (total 40 points) & \\
None & 40 \\
Mild, occasional & 30 \\
Moderate, daily & 20 \\
Severe, almost always present & 0
\end{tabular}

Function (total 50 points)

Limitation of activity or requirement of support

No limitations, no support

No limitation of daily activities, limitations of recreational activities, no support

Limited daily and recreational activities, cane

Severe limitation of daily and recreational activities, walker, 0 crutches, wheelchair, brace

Maximal walking distance, blocks

More than 6

4-6

$1-3$

Less than 1

2

Walking surfaces

No difficulty on any surface

Some difficulty on uneven terrain, stairs, inclines, ladders

Severe difficulty on uneven terrain, stairs, inclines, ladders

Gait abnormality

None, slight 0

Obvious

Marked

Sagittal motion (flexion plus extension; degrees) 4

Normal or mild restrictions (30 or more) $\quad 8$

Moderate restriction (15 to 29) 4

Severe restriction (less than 15) 0

Hindfoot motion (inversion plus eversion)

Normal or mild restriction $(75-100 \%$ of normal) 0

Moderate restriction (25-74\% of normal) 6

Marked restriction (less than $25 \%$ of normal) 3

Ankle/hindfoot stability (anteroposterior, varus-valgus)

Stable

Definitely unstable

Alignment (total 10 points)

Good, plantigrade foot, ankle-hindfoot well aligned

Fair, plantigrade foot, some degree of ankle-hindfoot malalignment observed, no symptoms

Poor, nonplantigrade foot, severe malalignment, symptoms

0

operation (nonsignificant). The mean plantar flexion range was $27 \pm 5^{\circ}$ before and $30 \pm 6^{\circ}$ after operation (nonsignificant). There was no evidence of instability in any of the 16 ankles/hindfeet examined and none complained of numbness. The average AOFAS clinical scale improved from a value of 36 preoperatively to a value of 78 at latest follow-up $(P<0.05)$. 

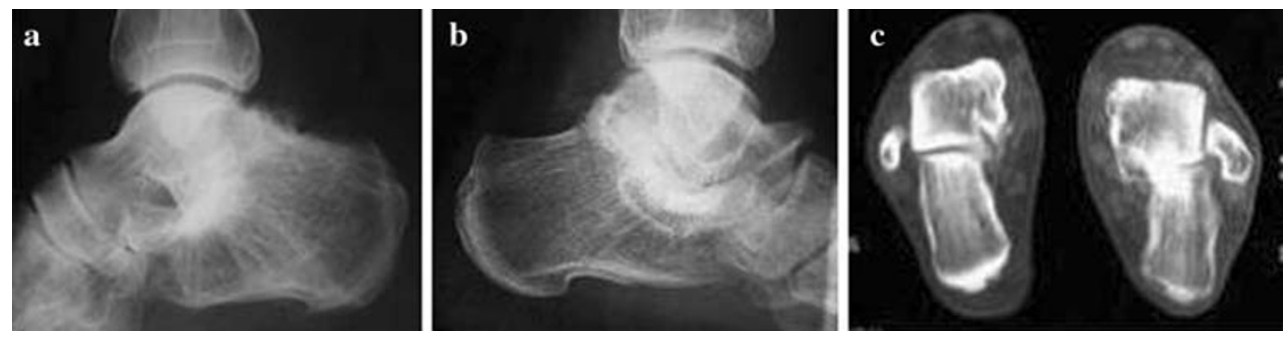

Fig. 2 a and b Radiographic evidence of union at the arthrodesis site on the lateral X-ray. c Coronal CT cuts at the level of the posterior subtalar facet (notice the medial placement of the tunnel and graft in order to correct hindfoot varus)

In the current study, union was achieved in 15 of the 16 feet (94\% union rate). At 4 months follow-up, 13 feet had partial union or a union bar visible on the lateral or Broden views and 2 feet were fully united (Fig. 2). There was one case of radiographic nonunion in our series with a visible radiolucency at the talar-graft interface. Despite the nonunion, this patient achieved a good AOFAS clinical score. There were measured increases in calcaneal inclination: calcaneal pitch angle $\left(6.25 \pm 8.3^{\circ}, P<0.07\right)$ and lateral talocalcaneal angle $\left(7.42 \pm 10.2^{\circ}, P<0.08\right)$. At latest follow-up, only 2 patients had evidence of arthritis of adjacent joints, mainly the talonavicular, with mild joint space narrowing, subchondral sclerosis, marginal osteophytes, and minor subchondral cysts. These two patients had a long time interval between fracture and arthrodesis, 4 and 5 years, respectively, with evidence of arthritis of adjacent joints evolving even on the preoperative radiographs. Good clinical results were achieved in both patients.

\section{Complications}

Transient tendonitis of the Achilles occurred in one patient. Radiographic nonunion at the talar-graft interface was found in one patient with a sagittal fracture gap and depression of the upper os calcis (Fig. 3), the site where the graft should have been inserted. Although this patient had good clinical results at latest follow-up, his case demonstrated the importance of fracture geometry in the decision-making for choosing the fusion method.

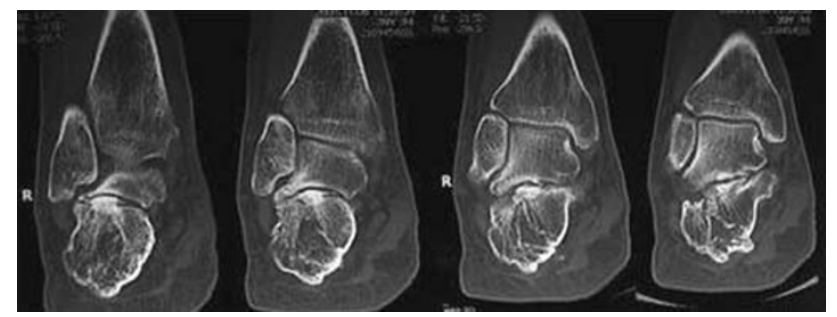

Fig. 3 Coronal CT cuts showing nonunion at the talar-graft interface and sagging of the graft in the longitudinal fracture gap
Alternative techniques might be better adopted in patients with a sagittal fracture gap and depression.

\section{Discussion}

Several authors have reported good results in the management of calcaneal fractures with extensive comminution of the posterior articular facet by primary subtalar fusion. Wilson [25] had 73 percent good results in patients who returned mostly to heavy work. Gallie [12] reported fifty patients with good results. Harris [15], using the same method of fusion, had 34 out of 35 patients back to work in 6 months. Geckeler [13] using the posterior approach for less deformed fractures and the lateral approach for the more deformed ones had his 27 patients back to work in 6 months, $70 \%$ in full work and $30 \%$ in light work. Dick [8] reported that all ten patients followed up had returned to work within six and a half months. Hall and Pennal reported that within an average time of six and a half months, $93 \%$ had returned to full employment and $81 \%$ had returned to the same job they had had at the time of their injury [14].

In this series, a standardized protocol was adopted to achieve fusion using a minimally invasive surgical technique that avoids hardware complications, allows immediate weight bearing, and still helps to correct the hindfoot deformity. The efficacy of the current treatment protocol was demonstrated by the fusion rate of $94 \%$ and the significant improvement of the AOFAS scores.

The fracture-to-arthrodesis interval was different in the various reported series. Dick waited until the swelling had subsided after rest, pressure bandaging, and elevation [8]. Conn treated his patients in traction for 5 weeks, and Wilson for not more than two [19, 25]. Harris, after manipulating the fracture, made his decision regarding operation from the radiographs, and the operation was performed soon thereafter [15] Lindsay and Dewar included all patients operated upon within 12 months of the injury [19]. Noble believed that posterior subtalar fusion should be undertaken between two and four weeks after injury in order to allow early return of satisfactory 
function, thus avoiding the damaging psychological and economic effects of delayed fusion or conservative care [21].

Experience in conservative management of the os calcis fracture has shown that better results are achieved with early joint movement. Pain and stiffness after injury is associated with unsatisfactory results; and with this in mind, primary fusion has been recommended [14]. Most of our patient presented with late sequelae of a fractured os calcis. The mean fracture-to-fusion interval was $2( \pm 1.6)$ years ranging from 6 months to 6 years post fracture. Despite this range, we noted that the shorter the interval, the better the post fusion AOFAS clinical score. We agree with advocates of early fusion that better clinical outcomes can be achieved before evident subtalar arthritis and stiffness become well established and arthritis of neighbouring joints become manifest. Nevertheless, in fractures with significant comminution, a reasonable time interval should be allowed for the fracture fragments to settle and provide a stable platform for the arthrodesis.

Gallie [12] first described the posterior approach to subtalar joint arthrodesis with insertion of bone block from the tibia without fixation in 1943. Carr et al. [4] later modified the technique by using an iliac crest graft and fully threaded screw fixation, this technique being similarly advocated by others $[1,3,4]$. However, some authors have also used either partially threaded or fully threaded screws placed with a lag technique for definitive fixation $[5,6,22]$. Different hardware configurations have been described to transfix the arthrodesis; this includes several single screw configurations and double parallel or divergent screws [9]. There are advantages and disadvantages described for each technique. Gable et al. cited a preference for placement of the screw from the posterior calcaneal tuberosity because it eliminates potential problems associated with screw placement from the dorsal neck of the talus into the calcaneum. The talar neck approach may cause anterior ankle impingement or a stress riser at the neck of the talus that would necessitate hardware removal. This approach also can jeopardize the blood supply to the talus, causing avascular necrosis of the talar head [11, 18]. In addition, Hutchinson [16] reported the advantage of increased density in the cancellous bone of the talus when compared with the calcaneum, theoretically offering more compression strength with the posterior calcaneal screw fixation approach.

A commonly cited disadvantage of the posterior approach is the need for fixation removal because of softtissue irritation over the prominent screw head [5, 18]. Mann et al. [20] reported that 33\% required fixation removal because of symptomatic fixation devices with this method of subtalar joint arthrodesis. Easley et al. [10] reported that $36(20 \%)$ of 184 patients in their study required hardware removal because of painful prominent screw heads over the posterior calcaneum.

The "no hardware" minimally invasive technique described in this study avoids all the above-mentioned disadvantages of implant placement from either approach, in addition to allowing immediate weight bearing as a consequence of the inherent stability of the arthrodesis. The fusion bed was not jeopardized by an implant providing better biology and more surface area for fusion, the risk of infection was reduced, and the length of hospital stay was kept to a minimum (mean 1.3 days \pm 0.4 ). In addition, the technically demanding screw insertion was avoided, and hence the operative time and radiation exposure reduced - an advantage that justifies the adage "simplicity is the ultimate sophistication". In contrast, other authors have advocated a high technology approach and conclude that the use of computer-assisted surgery for subtalar arthrodesis decreases the number of suboptimal guide wire passes and fluoroscopic radiation exposure while maintaining a screw placement accuracy rate that is equivalent to the rate associated with conventional subtalar arthrodesis [9].

Attention is raised to the fracture geometry where, in the presence of a sagittal gap and depression of the upper os calcis, the graft might subside and sag inferiorly within the os calcis as encountered in the one case of radiographic nonunion in this series. In this, alternative techniques should be sought to provide stability across the fusion site. Chan and Alexander [5] have also reported 1 surgical failure where a single tricortical interpositional bone graft completely disappeared into the osteoporotic calcaneum. This led to a two-technique modification: first, 2 interpositional grafts were used versus one and, second, partially threaded screws versus fully threaded ones. They believed that the greater surface area of the double graft would prevent loss of heel height and the partially threaded screw would hold the position through compression that would facilitate graft incorporation [22].

Autogenous iliac crest has been the preferred graft for posterior bone-block distraction arthrodesis [1-3, 5] Furthermore, in those patients who need substantial distraction for optimal alignment, the size of available and safe distal tibial graft can be insufficient as the sole source of graft. Baravarian [2] reported on 8 of 12 patients who had tricortical allograft for the index procedure. No difference in the healing time with allogenic and autogenous bone grafting was observed. Clare et al. [6] used the excised lateral wall fragment as an autograft bone block. Trnka et al. [24] used either tricortical iliac bone block or a femoral head contoured to fit the space. Pollard and Schuberth [22] found no difference in the union rate of allograft when compared to autograft and hence advised use of a tricortical iliac crest allograft for the main 
structural graft component combined with a distal tibial bone graft as the main biologic component.

In the current study, autograft was obtained from the posterior iliac crest for three reasons. First, the posterior iliac graft was easily obtained from a prone patient. This position is also convenient for the posterolateral approach to the subtalar joint as well, additionally providing easier access for fluoroscopic imaging. Second, the posterior ileum provides a graft with better and stronger bone quality when compared with the anterior ileum. Third, the pain and morbidity are less when compared with anterior iliac grafting. No patients in our series had significant pain or morbidity related to posterior iliac graft whether in the early postoperative period or at latest follow-up. The conically shaped cortico-cancellous wedge served to distract the fusion site along the prepared tunnel, restore the heel height, calcaneal inclination $(P<0.07)$, and hindfoot alignment $(P<0.08)$. Medialisation of the tunnel for graft placement was the key point in the successful correction of hindfoot varus in most of the cases.

In conclusion, we found the minimally invasive posterolateral percutaneous approach for subtalar arthrodesis using posterior iliac bone graft, followed by immediate weight bearing in a cast boot extremely useful in the management of sequelae of os calcis fractures. Excellent clinical and radiographic results could be achieved and hindfoot deformity could be corrected with such approach. The technique is simple, less traumatic, less time-consuming, and hardware related complications could be avoided. Further studies are needed to solve the problem of graft subsidence and concomitant nonunion in cases with persisting sagittal fracture gap.

Acknowledgments We would like to express our gratitude and respect to the persons whose cadavers were utilized by the visible human server and all the staff members of the EPFL (École Polytechnique Fédérale de Lausanne) who participated in this outstanding project that served us to construct cadaveric dissections electronically without having to visit the laboratory.

\section{References}

1. Amendola A, Lammens P (1996) Subtalar arthrodesis using interposition iliac crest bone graft after calcaneal fracture. Foot Ankle Int 17:608-614

2. Baravarian B (2004) Block distraction arthrodesis for the treatment of failed calcaneal fractures. Clin Podiatr Med Surg 21:241-250

3. Bednarz PA, Beals TC, Manoli A (1997) Subtalar distraction bone block fusion: an assessment of outcome. Foot Ankle Int 18:785-791
4. Carr JB, Hansen ST, Benirschke SK (1988) Subtalar distraction bone block fusion for late complications of os calcis fractures. Foot Ankle 9:81-86

5. Chan SC, Alexander IJ (1997) Subtalar arthrodesis with interposition tricortical iliac crest graft for late pain and deformity after calcaneum fracture. Foot Ankle Int 18:613-615

6. Clare MP, Lee WE, Sanders RW (2005) Intermediate to longterm results of a treatment protocol for calcaneal fracture malunions. J Bone Joint Surg 87A:963-973

7. Dahm DL, Kitaoka HB (1998) Subtalar arthrodesis with internal compression for post-traumatic arthritis. J Bone Joint Surg 80$\mathrm{B}(1): 134-138$

8. Dick IL (1953) Primary fusion of the posterior subtalar joint in the treatment of fractures of the calcaneum. J Bone Joint Surg 35-B(3):375-380

9. Easley M, Chuckpaiwong B, Cooperman N et al (2008) Computer-assisted surgery for subtalar arthrodesis: a study in cadavers. J Bone Joint Surg 90-A(8):1628-1636

10. Easley ME, Trnka HJ, Schon LC, Myerson MS (2000) Isolated subtalar arthrodesis. J Bone Joint Surg 82-A(5):613-624

11. Gable SJ, Bohay DR, Manoli A (1995) Aiming guide for accurate placement of subtalar joint screws. Foot Ankle Int 16:238-239

12. Gallie WE (1943) Subastragalar arthrodesis in fractures of the os calcis. J Bone Joint Surg 25A:731-736

13. Geckeler EO (1950) Comminuted fractures of the Os Calcis. Arch Surg 61:469

14. Hall MC, Pennal GF (1960) Primary subtalar arthrodesis in the treatment of severe fractures of the calcaneum. J Bone Joint Surg 42-B(2):336-343

15. Harris RI (1946) Fractures of the Os Calcis. Ann Surg 124:1082

16. Hutchinson B (2000) Current concepts in triple arthrodesis. Clin Podiatr Med Surg 17:1-17

17. Kitaoka HB, Alexander IJ, Adelaar RS et al (1994) Clinical rating systems for the ankle-hind foot, midfoot, hallux, and lesser toes. Foot Ankle Int 15:349-353

18. Lehnert B, Gosch C, Sims G (2004) A plantar approach for fixation of subtalar joint arthrodesis. J Foot Ankle Surg 43(1):67-69

19. Lindsay WR, Dewar FP (1958) Fractures of the Os Calcis. Am J Surg 95:555

20. Mann RA, Beaman DN, Horton GA (1998) Isolated subtalar arthrodesis. Foot Ankle 19:452-461

21. Noble J, McQuillan WM (1979) Early posterior subtalar fusion in the treatment of fractures of the os calcis. J Bone Joint Surg 61B(1):90-93

22. Pollard JD, Schuberth JM (2008) Posterior bone block distraction arthrodesis of the subtalar joint: a review of 22 cases. J Foot Ankle Surg 47(3):191-198

23. Russotti GM, Cass JR, Johnson KA (1988) Isolated talocalcaneal arthrodesis: a technique using moldable bone graft. J Bone Joint Surg 70-A:1472-1478

24. Trnka HJ, Easley ME, Lam PW et al (2001) Subtalar distraction bone block arthrodesis. J Bone Joint Surg 83B:849-854

25. Wilson PD (1933) Fractures and dislocations of the tarsal bones. South Med J 26:833

26. Zion I, Shabat S, Marin L et al (2003) Subtalar distraction arthrodesis using a ramp cage. Orthopedics 26:1117-1119 